BOEKBR, W. \& HAEFNER, H. (1973) Gewalttaten Geistesgestoerter. Eine psychiatrische-epidemiologische Untersuchung in der Bundesrepublik Deutschland. Berlin: Springer.

Curfel, B. J. (1992) Prevalence estimates of substance abuse in schizophrenia and their correlates. Journal of Nervous and Mental Diseases, 180, 589-593.

SoYkA, M. (1993) Substance abuse and dependency as a risk factor for delinquency and violent behavior in schizophrenic patients how strong is the evidence? Journal of Clinical Forensic Medicine, 1, 3-7

-, Albus, M., Fnnelli, A., et al (1993) Prevalence of alcohol and drug abuse in schizophrenic inpatients. European Archives of Psychiatry and Clincial Neuroscience, 242, 362-372.

Psychiatric Hospital

University of Munich

Nussbaumstrasse 7

80336 Munich, Germany

\section{Psychopathology and 'ecstasy'}

SIR: Maguire et al (BJP, September 1994, 165, 391-395) have presented an interesting, if small, study comparing psychiatric symptoms in patients who have taken MDMA ('ecstasy') with a control group who showed no evidence of drug use. However, having presented useful data they draw conclusions that cannot be sustained by their evidence. The authors appear to believe that the symptoms displayed by the 13 patients had a causal link with the use of MDMA. They report that the symptoms displayed by the eight psychotic patients did not differ from the matched controls. Four of the eight psychotic patients had discontinued MDMA use four or more weeks prior to psychiatric presentation, and one patient had discontinued 26 weeks earlier. There was no clear relationship between MDMA use and the onset of symptoms. Little information is offered as to the course that the patients' illnesses took, other than in case 8 where it would appear that symptoms continued irrespective of MDMA use.

We suggest that, from the available evidence, it is equally likely that these patients were suffering from one of the major psychoses. While it is conceivable that MDMA use precipitated or coloured the symptoms, a firm causal link is not sustainable. Use of drugs of abuse, including MDMA, has become widespread in the general population and the coincidence of a psychotic breakdown with drug-taking is bound to happen frequently. It would be dangerous to draw the conclusion that these disorders should necessarily be classified or managed any differently to other psychotic disorders.

The Clunis Report (Ritchie et al, 1994) describes in detail a case where a history of supposed drug use was uncritically taken as an explanation for the persistently abnormal mental state of a man suffering from schizophrenia. This contributed, albeit in a minor way, to the inadequate management of a man who went on to kill. The case illustrates the potential dangers of mistaken attribution of psychotic states to drug use. It is important that the published literature should not exaggerate the strength of the evidence for putative causal relationships to the exclusion of other plausible explanations.

Rrrchin, J., Dick, D. \& Linghum, R. (1994) The Report of the
Enquiry into the Care and Treatment of Christopher Clunis. London: HMSO.

R. G. POOLE

C. J. BRABBINS

Windsor Mental Health Resource Centre Liverpool L8 $7 L F$

\section{Cognitive therapy}

SIR: We would like to bring to your attention a significant omission from Scott's otherwise excellent article on cognitive-behavioural therapy or CBT (BJP, July 1994, 165, 126-130). While she does justice to its application to mood and anxiety disorders, she makes no mention of its application to eating disorders and bulimia nervosa in particular. This omission is surprising since the evidence supporting this specific application of CBT is at least as strong as in other areas, if not more so.

The rationale for using a cognitively-oriented treatment for bulimia nervosa arises from the prominence of these patients' extreme concerns about shape and weight and their likely role in the maintenance of the disorder (Wilson \& Fairburn, 1993). This application of CBT has been the subject of over 15 randomised controlled trials and their findings support its use (Fairburn et al, 1992). Once maintenance of change is taken into account, it is clear that the effects of CBT are superior to those of all other treatments studied, with the possible exception of interpersonal psychotherapy (Fairburn et al, 1993a). Between a half and two-thirds of patients make a full and lasting recovery and many of the remainder are much improved.

A detailed manual describing CBT for bulimia nervosa has been available for many years - the latest version was published in 1993 (Fairburn et al, 1993b). The treatment has been adapted to suit patients with anorexia nervosa (Garner \& Bemis, 1985) but this adaptation has yet to be adequately evaluated. The role of CBT in the 